\title{
Constraints on Newton's constant from cosmological observations
}

\author{
Ke Wang ${ }^{1, \mathrm{a}}$, Lu Chen ${ }^{2, \mathrm{~b}}$ \\ ${ }^{1}$ Institute of Theoretical Physics and Research Center of Gravitation, Lanzhou University, Lanzhou 730000, China \\ 2 School of Physics and Electronics, Shandong Normal University, Jinan 250014, China
}

Received: 29 April 2020 / Accepted: 10 June 2020 / Published online: 25 June 2020

(C) The Author(s) 2020

\begin{abstract}
Newton's constant has observational effects on both the CMB power spectra and the light curves of SNIa. We use Planck data, BAO data and the SNIa measurement to constrain the varying Newton's constant $G$ during the $\mathrm{CMB}$ epoch and the redshift ranges of PANTHEON samples, and find no evidence indicating that $G$ is varying with redshift. By extending the $\Lambda \mathrm{CDM}$ model with one free parameter $G$, we get $G=\left(6.65635_{-0.18560}^{+0.18766}\right) \times 10^{-11} \mathrm{~m}^{3} \mathrm{~kg}^{-1} \mathrm{~s}^{-2}$ and $H_{0}=67.62_{-1.25}^{+1.24} \mathrm{~km} \mathrm{~s}^{-1} \mathrm{Mpc}^{-1}$ at $68 \% \mathrm{CL}$ from Planck+BAO+uncalibrated PANTHEON. The results show the value of $G$ is consistent with CODATA 2018, but the $H_{0}$ tension can't be solved in this way.
\end{abstract}

\section{Introduction}

Newton's gravitational constant is treated as a constant both in Newton's gravitational theory and general relativity. Over one hundred years after Newton proposed its definition, Henry Cavendish measured the value of $G=6.754 \pm 0.041 \times$ $10 \mathrm{~N} \cdot \mathrm{m}^{2} / \mathrm{kg}^{2}$ with torsion scale experiment. Since then, kinds of methods are used to determine Newton's constant more precisely. In 2019, the Committee on Data for Science and Technology (CODATA) gives its recommended value of $G=6.67430 \times 10^{-11} \mathrm{~m}^{3} \mathrm{~kg}^{-1} \mathrm{~s}^{-2}$ (named CODATA 2018) and the standard uncertainty is $1.5 \times 10^{-15} \mathrm{~m}^{3} \mathrm{~kg}^{-1} \mathrm{~s}^{-2}$, which means $2.2 \times 10^{-5}$ relative uncertainty. In the laboratory, cold atom interferometry is also used to detect Newton's constant [1]. In cosmology, the cosmic microwave background (CMB) [2-7], big bang nucleosynthesis (BBN) [810], type Ia supernovae (SNIa) [11-15] and gravitational waves $[14,16]$ can provide different measurements of Newton's constant at corresponding epochs of our universe. Obviously, there is a problem whether Newton's constant is always a constant really or not. Theoretically, it is acceptable to be

\footnotetext{
${ }^{a}$ e-mail: wangkey@1zu.edu.cn

be-mail: chenlu@mail.itp.ac.cn (corresponding author)
}

both time- or space-dependent in some theories of modified gravity [17-19]. For example, the scalar-tensor theories predict a time-dependent $G$. The cosmological observation provides a method to study the Newton's constant varying with redshift.

Any change in Newton's constant have influence on the expansion history of our universe, especially at the redshift of recombination, which leaving a footprint on the $\mathrm{CMB}$ power spectra. Combing the precise observation of Planck collaboration [20], Newton's constant during the CMB epoch can be restricted. SNIa measurement, as the standard candles, are usually used to study the accelerated expansion, too. Newton's constant affects its peak luminosity through the Chandrasekhar mass by $M_{\mathrm{Ch}} \propto G^{-3 / 2}$ mostly. The latest SINa data, PANTHEON samples [21], detected the light curves of 1048 SNIa covering the redshift range $0<z<2.3$ and provides a way to limit Newton's constant at low redshift. Therefore, we constrain the varying Newton's constant with the CMB power spectra and the SNIa peak luminosity and probe its dynamics.

Besides, the Hubble constant $H_{0}$ indicates the expansion of the universe directly. Plank collaboration claimed $H_{0}=$ $67.4 \pm 0.5 \mathrm{~km} \mathrm{~s}^{-1} \mathrm{Mpc}^{-1}$ after its final data release [20]. However, the SHOES project yielded the best estimate as $H_{0}=74.03 \pm 1.42 \mathrm{~km} \mathrm{~s}^{-1} \mathrm{Mpc}^{-1}$ (named R19), which is $4.4 \sigma$ different from Planck [22]. $H_{0}$ tension may result from systematic errors of measurements. Errors of both the SH0ES and Planck data are studied in recent years [23-26]. However, other alternative data show a similar discrepancy with the CMB measurement. Another possibility is that $H_{0}$ tension implies new physics beyond the $\Lambda \mathrm{CDM}$ model. Some experts attempt to solve the tension by extending the base $\Lambda \mathrm{CDM}$ model simply, such as the dark energy (DE) equation of state $w$, the effective number of relativistic species $N_{\text {eff }}$, the total mass of neutrinos $\Sigma m_{v}$, and so on [27-32]. Moreover, modifying early universe physics and changing late-time cosmology influence Hubble constant significantly. From this view, dynamical DE [33-38], early DE [39-42], interacting 
DE [43-46], dark radiation [47,48], scalar fields [49,50], and many other components are considered to solve $H_{0}$ tension. Unfortunately, it has not been well solved till now. Owing to the effect of Newton's constant on the Hubble parameter $H(z)$, we expect a solution of $H_{0}$ tension by modifying $G$. Recently, Ref. [51] has discussed the varying $G$ in the scalar-tensor theory of gravity, which influences the expansion history of our universe before recombination epoch. They gave the result of $H_{0}=69.2_{-0.75}^{+0.62} \mathrm{~km} \mathrm{~s}^{-1} \mathrm{Mpc}^{-1}$. And Ref. [52] also finds a larger value for $H_{0}$ by an evolving gravitational constant. Here, we simply set $G$ as a free parameter in the base $\Lambda \mathrm{CDM}$ model to enlarge the value of $H_{0}$.

This paper is organized as follows. In Sect. 2.1, we rescale Newton's constant by introducing $\lambda_{2}$ and sketch out its influence on the CMB power spectra. In Sect. 2.2, the effect of Newton's constant on the peak luminosity of SNIa is presented. We show our results in Sect. 3. We turn to CAMB and the Markov Chain Monte Carlo (MCMC) package CosmoMC [53]. The CMB data, BAO data and the SNIa measurement are used to constrain the varying Newton's constant in Sect. 3.1. Then the data combination of CMB, BAO and uncalibrated SNIa is applied to study the $H_{0}$ tension with a constant G in Sect. 3.2.

Finally, a brief summary and discussion are included in Sect. 4.

\section{Effects of Newton's gravitational constant on cosmological observations}

To weigh the effects of Newton's gravitational constant on some cosmological observations, we rescale $G_{N}=6.6738 \times$ $10^{-11} \mathrm{~m}^{3} \mathrm{~kg}^{-1} \mathrm{~s}^{-2}$ with several dimensionless parameter $\lambda_{i}$, $i=0,1,2$, then the new definition of Newton's constant is

$G= \begin{cases}\lambda_{0}^{2} G_{N}, & \text { for } z<0.1 \\ \lambda_{1}^{2} G_{N}, & \text { for } 0.1 \leq z<2.3 \\ \lambda_{2}^{2} G_{N}, & \text { for } 2.3 \leq z\end{cases}$

Here, $G$ indicates the effective values of Newton's constant for each bins actually. Then the Friedmann equation is

$\mathcal{H}^{2}=\left(\frac{\dot{a}}{a}\right)^{2}= \begin{cases}\frac{8 \pi}{3} a^{2} \lambda_{0}^{2} G_{N} \rho, & \text { for } z<0.1 ; \\ \frac{8 \pi}{3} a^{2} \lambda_{1}^{2} G_{N} \rho, & \text { for } 0.1 \leq z<2.3 \\ \frac{8 \pi}{3} a^{2} \lambda_{2}^{2} G_{N} \rho, & \text { for } 2.3 \leq z\end{cases}$ where $\mathcal{H}$ is the Hubble rate , $a$ is the scale factor, $\rho$ is the total energy density in the universe and overdot means the differentiation over the conformal time $\tau$. When we rescale $\tau$ as

$d \tau \rightarrow \lambda_{i} d \tau=\frac{\lambda_{i} d t}{a}=\frac{d a}{a^{2} \sqrt{8 \pi / 3 G_{N} \rho}}$

the integrand of cosmic distances are independent of $\lambda_{i}$. Therefore, we cannot use the cosmic distances only, like the BAO measurements with Eisenstein's baryon drag epoch $z_{d}$ [54], to constrain Newton's gravitational constant, but we can turn to other non-gravity interactions to constrain $G$.

\subsection{Effects of Newton's gravitational constant on CMB during the recombination}

To the first order, the Boltzmann equations of the baryons and photons in the conformal Newtonian gauge reads

$\dot{\delta}_{\gamma}=-\frac{4}{3} \theta_{\gamma}+4 \dot{\phi}$

$\dot{\theta}_{\gamma}=\frac{1}{4} k^{2} \delta_{\gamma}+k^{2} \psi+a n_{e} \sigma_{T}\left(\theta_{b}-\theta_{\gamma}\right)$,

$\dot{\delta}_{b}=-\theta_{b}+3 \dot{\phi}$

$\dot{\theta}_{b}=-\frac{\dot{a}}{a} \theta_{b}+c_{s}^{2} k^{2} \delta_{b}+\frac{4 \bar{\rho}_{\gamma}}{3 \bar{\rho}_{b}} a n_{e} \sigma_{T}\left(\theta_{\gamma}-\theta_{b}\right)+k^{2} \psi$,

where $\delta=\delta \rho / \bar{\rho}$ is the density fluctuation, $\theta$ is the velocity perturbation for a given mode $k, \phi$ and $\psi$ represent the scalar mode of metric perturbations, $\sigma_{T}$ is the crosssection of Thomson scattering, $n$ is the number density and $\left(c_{s}^{2}\right)^{-1}=3\left(1+\frac{3 \bar{\rho}_{b}}{4 \bar{\rho}_{\gamma}}\right)$ is the sound speed of baryons. The subscript $e$ represents electrons, $\gamma$ is photons and $b$ means baryons. If the two Thomson scattering terms in Eq. (4) are ignored, the replacement of $\tau$ by $\lambda_{i} \tau$ must accompanies a replacement of $k$ by $k / \lambda_{i}$ for keeping Eq. (4) (or $\mathrm{CMB}$ observations) unchanged. Therefore, the transformation of $\tau \rightarrow \lambda_{i} \tau$ also cannot be observed through perturbations because the transformation of $k \rightarrow k / \lambda_{i}$ can be compensated by adjusting the scalar spectral index $n_{s}$ appropriately if large-scale structure clustering measurements are not considered. Fortunately, there exists Coulomb interaction. So the only way that $\lambda_{i}$ influences the CMB anisotropy spectrum is affecting the number of free electrons $n_{e}$ during the recombination epoch, hence the ionization fraction $x_{e}=n_{e} / n_{\mathrm{H}}=x_{p}+x_{\mathrm{HeII}}$ during the same epoch. Here, $n_{\mathrm{H}}$ is the total number density of $\mathrm{H}$ nuclei, $x_{p}$ is the ionization fraction of $\mathrm{H}$ and $x_{\mathrm{HeII}}$ presents that of He. According to Ref. [55], the modified evolution of $x_{p}$ and $x_{\text {HeII }}$ can be obtained by solving the following ordinary differential equations (ODEs) 


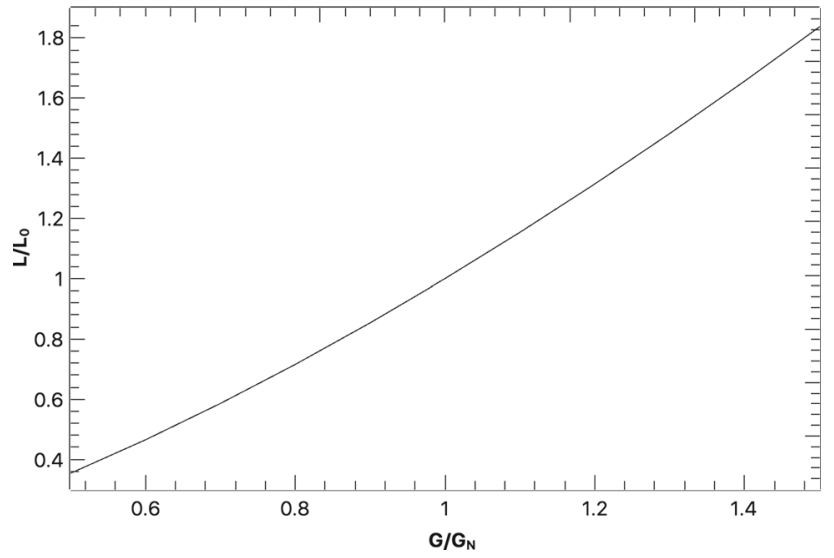

Fig. $1 L / L_{0}$ as a function of $G / G_{N}$

$$
\begin{aligned}
\frac{d x_{p}}{d z} & =\frac{f_{1}\left(x_{e}, x_{p}, n_{\mathrm{H}}, T_{\mathrm{M}}\right)}{H\left(\lambda_{2}, z\right)(1+z)}, \\
\frac{d x_{\mathrm{HeII}}}{d z} & =\frac{f_{2}\left(x_{e}, x_{\mathrm{HeII}}, n_{\mathrm{H}}, T_{\mathrm{M}}\right)}{H\left(\lambda_{2}, z\right)(1+z)}, \\
\frac{d T_{\mathrm{M}}}{d z} & =\frac{f_{3}\left(x_{e}, T_{\mathrm{M}}, T_{\mathrm{R}}\right)}{H\left(\lambda_{2}, z\right)(1+z)}+\frac{2 T_{\mathrm{M}}}{(1+z)},
\end{aligned}
$$

where $T_{\mathrm{M}}$ (or $T_{\mathrm{R}}$ ) is the matter (or radiation) temperature, the specific expressions of $f_{1}, f_{2}$ and $f_{3}$ are given in Ref. [55]. From above ODEs, we can find that $x_{e}$ evolves slower for $\lambda_{2}>1$, hence a latter photo-decoupling time $z_{*}$ and baryon drag epoch $z_{d}$. Therefore, we can use the data combination of CMB and BAO measurements to constrain $\lambda_{2}$.

\subsection{Effects of Newton's gravitational constant on the SNIa}

Since the effects of $\lambda_{2}$ on CMB is confined to $n_{e}$ during the recombination epoch, there is a possibility that the deviation of Newton's constant from $G_{N}$ is not equal to $\lambda_{2}$ at other different epochs. Therefore, it's necessary to introduce new parameters to quantify the potential deviation from $G_{N}$ after the recombination epoch, especially if the constraints on the deviation are not from CMB observations.

Newton's constant influences the light curve of SNIa via the Chandrasekhar mass $M_{\mathrm{Ch}} \propto G^{-3 / 2}$ mainly. If the Newton's constant $G$ increases, the peak luminosity of light curve $L$ raises and its width drops [11]. In this section, we introduce a new parameter $\lambda_{1}^{\prime}=L / L_{0}$ to quantify the deviation of $L$ from $L_{0}$ resulting from the deviation of $G$ from $G_{N}$. Figure 1 shows a sketch of $\lambda_{1}^{\prime}=L / L_{0}$ as a function of $G / G_{N}$, from which we can derive $G / G_{N}$ from any given $\lambda_{1}^{\prime}$. The two parameters are almost linearly. In other words, the derivation of $\lambda_{1}^{\prime}$ from 1 is nearly equivalent to the difference between $G$ and $G_{N}$. If we use the final redshifts, corrected magnitudes $\mu+M_{B}$ and the host galaxy mass $M_{\text {host }}$ of PANTHEON samples [21] to constrain cosmological parameters, the combination of $\mu+M_{B}$ can be related to $\lambda_{1}^{\prime}$ as

$$
\begin{aligned}
\mu+M_{B}= & 5 \log _{10}\left[\frac{d_{L}}{\mathrm{Mpc}}\right]+25+M_{B}^{1}-2.5 \log _{10} \lambda_{1}^{\prime}+\Delta_{M} \\
= & 5 \log _{10}\left[\frac{d_{L}}{\mathrm{Mpc}}\right]+25 \\
& \quad+M_{\odot}-2.5 \log _{10}\left[\lambda_{1}^{\prime} L_{0} / L_{\odot}\right]+\Delta_{M}
\end{aligned}
$$

and $\Delta_{M}$ is related to $M_{\text {host }}$,
Table 1 The $68 \%$ limits for the cosmological parameters in two models for different purpose. Notice that $\lambda_{1}\left(\lambda_{1}^{\prime}\right)$ indicates $\lambda_{1}$ is a function of $\lambda_{1}^{\prime}$

\begin{tabular}{lll}
\hline & $\begin{array}{l}\text { Probing the } \\
\text { dynamics of } G \text { with } \\
\text { CMB, BAO and } \\
\text { SNIa }\end{array}$ & $\begin{array}{l}\text { Solving the } H_{0} \text { tension } \\
\text { with CMB, BAO and } \\
\text { uncalibrated SNIa }\end{array}$ \\
\hline$\Omega_{b} h^{2}$ & $0.02236 \pm 0.00016$ & $0.02237 \pm 0.00076$ \\
$\Omega_{c} h^{2}$ & $0.1197 \pm 0.0010$ & $0.1189 \pm 0.0035$ \\
$100 \theta_{\mathrm{MC}}$ & $1.04195_{-0.00144}^{+0.00143}$ & $1.04119_{-0.00206}^{+0.00208}$ \\
$\tau_{\mathrm{re}}$ & $0.055 \pm 0.007$ & $0.056 \pm 0.007$ \\
$\ln \left(10^{10} A_{s}\right)$ & $3.043 \pm 0.015$ & $3.047_{-0.015}^{+0.014}$ \\
$n_{S}$ & $0.9629 \pm 0.0064$ & $0.9665_{-0.0062}^{+0.0063}$ \\
$H_{0}\left[\mathrm{~km} \mathrm{~s}^{-1} \mathrm{Mpc}^{-1}\right]$ & $67.78 \pm 0.48$ & $67.62_{-1.25}^{+1.24}$ \\
$\lambda_{0}$ & 1 & \\
$\lambda_{1}$ & $\lambda_{1}\left(\lambda_{1}^{\prime}=1.003 \pm 0.015\right)$ & $0.999 \pm 0.014$ \\
$\lambda_{2}$ & $0.971_{-0.047}^{+0.043}$ & \\
$M_{B}^{1}$ & $-19.363 \pm 0.020$ & - \\
\hline
\end{tabular}




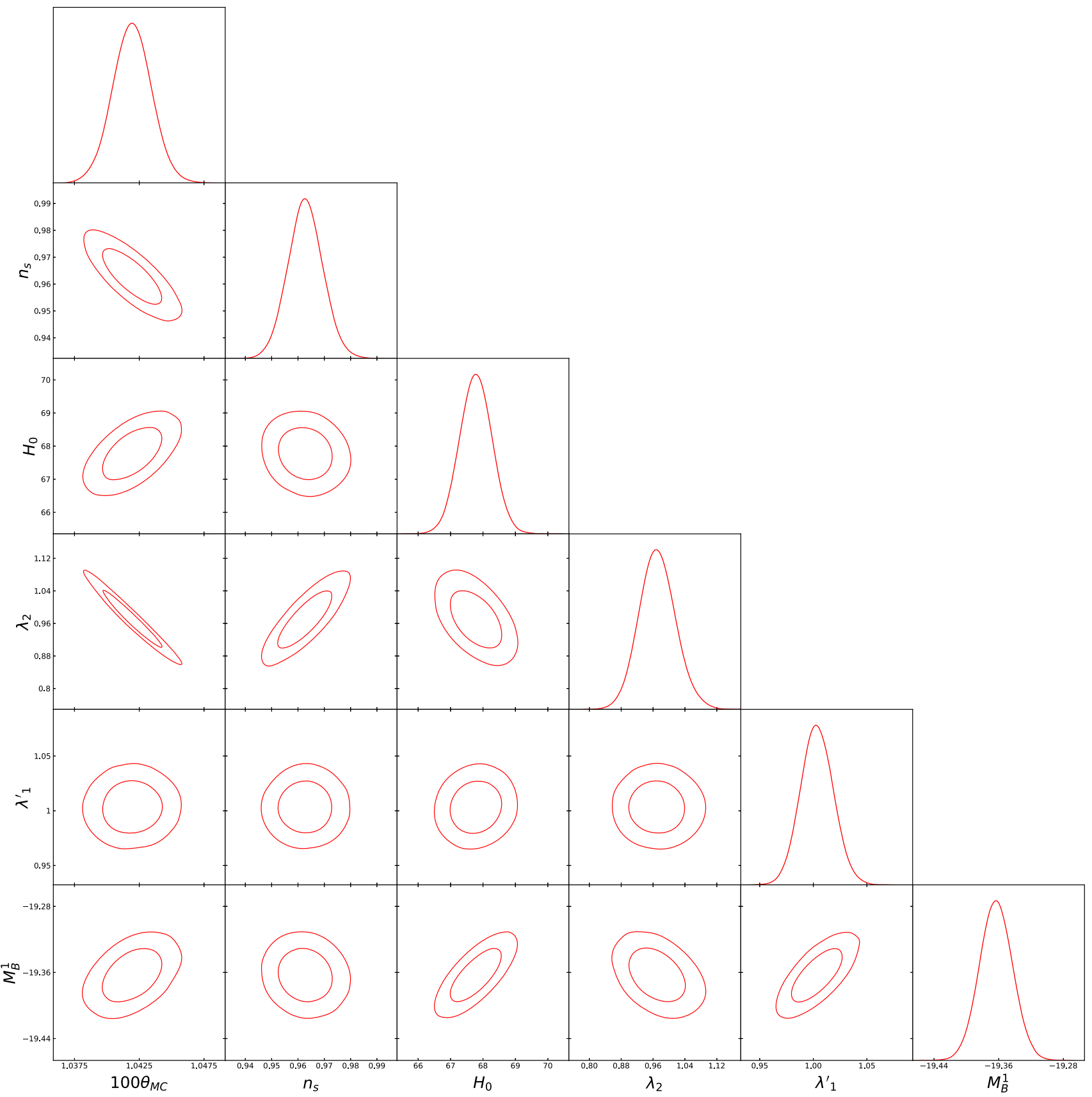

Fig. 2 The constraints on $\lambda_{1}^{\prime}, \lambda_{2}$ and $M_{B}^{1}$ from the data combination of CMB, BAO and SNIa. Also we present the constraints on $n_{s} H_{0}$ and $\theta_{\mathrm{MC}}$ which are affected most by the former three parameters

$\Delta_{M}= \begin{cases}0, & \text { for } M_{\text {host }}<10^{10} M_{\odot}, \\ -0.08 \mathrm{mag}, & \text { for } M_{\mathrm{host}} \geq 10^{10} M_{\odot} .\end{cases}$

Due to the degeneracy between $\lambda_{1}^{\prime}$ and $L_{0}$ (or $M_{B}^{1}$ ), we ignore the term of $-2.5 \log _{10} \lambda_{1}^{\prime}$ for $z<0.1$ and use samples at this redshift span to constrain $L_{0}$ (or $\left.M_{B}^{1}\right){ }^{1}$ Then samples from $z>0.1$ will be used to constrain $\lambda_{1}^{\prime}$.

\footnotetext{
${ }^{1}$ Since $L_{0}$ (or $M_{B}^{1}$ ) is defined with respect to $G_{N}$, it's convenient to set $\lambda_{0}=1$ to constrain $L_{0}$ (or $M_{B}^{1}$ ) directly.
}

\section{Results}

\subsection{Varying $\mathrm{G}$ with redshift}

Firstly, we consider an extension of $\Lambda$ CDM model with another two free parameters $\lambda_{1}^{\prime}$ (or $\lambda_{1}$ ) and $\lambda_{2}$ to probe the dynamics of $G$. Based on the previous discussion, $\lambda_{1}^{\prime}$ and $\lambda_{2}$ are used to measure the varying Newton's constant $G$ during the period of SNIa measurement $(z \sim$ 
$0.1-2.3)$ and the recombination epoch $(z \sim 1100)$ respectively. In summarize, the free parameters needed to be fitted are $\left\{\Omega_{b} h^{2}, \Omega_{c} h^{2}, 100 \theta_{\mathrm{MC}}, \tau_{\mathrm{re}}, \ln \left(10^{10}\right) A_{s}, n_{s}, \lambda_{1}^{\prime}, \lambda_{2}\right\}$. Here $\Omega_{b} h^{2}$ and $\Omega_{c} h^{2}$ are today's density of baryonic matter and cold dark matter respectively, $100 \theta_{\mathrm{MC}}$ is 100 times the ratio of the angular diameter distance to the large scale structure sound horizon, $\tau_{\mathrm{re}}$ is the optical depth, $n_{S}$ is the scalar spectrum index, and $A_{s}$ is the amplitude of the power spectrum of primordial curvature perturbations. We refer to CAMB and CosmoMC [53] and use the data combination of the latest $\mathrm{CMB}$ data released by the Planck collaboration in 2018, Planck 2018 TT,TE,EE+lowE+lensing [20], the BAO data including MGS [56], 6DF [57] and DR12 [58] and the PANTHEON sample consisting of 1048 SNIa measurements. The results are summarized in the first column of Table $1 . \lambda_{2}$ is $0.971_{-0.047}^{+0.043}$ and $\lambda_{1}^{\prime}$ is $1.003 \pm 0.015$ at $68 \%$ CL. According to Fig. 1, it indicates that the Newton's constant $G=G_{N}$ is still acceptable both during the recombination epoch and in the late-time universe till now. There is no evidence indicating the dynamic property of the Newton's constant. The Hubble constant $H_{0}$ reads $67.78 \pm 0.48 \mathrm{~km} \mathrm{~s}^{-1} \mathrm{Mpc}^{-1}$ at $68 \% \mathrm{CL}$, which is in agreement with the result $67.4 \pm 0.5 \mathrm{~km} \mathrm{~s}^{-1} \mathrm{Mpc}^{-1}$ of Planck 2018. The $68 \%$ limits for $M_{B}^{1}$ is $-19.363 \pm 0.020 \mathrm{mag}$, which is smaller than the previous constraint $-19.13 \pm$ 0.01 [14]. The triangular plot of $\lambda_{1}^{\prime}, M_{B}^{1}, \lambda_{2}, H_{0}, 100 \theta_{\mathrm{MC}}$ and $n_{s}$ is also shown in Fig. 2. $\lambda_{1}^{\prime}$ has positive correlation with $M_{B}^{1}$ as shown in Eq. (8), but it's almost independent of other parameters. By comparison, $\lambda_{2}$ is much more complicated. It has strong and negative relationship with $100 \theta_{\mathrm{MC}}$ and $H_{0}$ due to its affect on $z_{*}$. The correlation between $\lambda_{2}$ and $n_{s}$ results from the transformation of $k \rightarrow k / \lambda_{2}$.

\section{2 $H_{0}$ tension and constant $G$ with redshift}

Then, we try to solve the Hubble tension with a varying Newton's constant by consider an simple extension of $\Lambda \mathrm{CDM}$ model with another one free parameter $\lambda_{0}=$ $\lambda_{1}=\lambda_{2}$. We use the data combination of Planck 2018 TT,TE,EE+lowE+lensing, the BAO data (6DF, MGS and DR12) and uncalibrated PANTHEON sample. The results are shown in the second column of Table 1: $\lambda_{i}=0.999 \pm 0.014$ and $H_{0}=67.62_{-1.25}^{+1.24} \pm \mathrm{km} \mathrm{s}^{-1} \mathrm{Mpc}^{-1}$ at $68 \% \mathrm{CL}$. Our results indicate that Newton's constant $G=\left(6.65635_{-0.18560}^{+0.18766}\right)$ $\times 10^{-11} \mathrm{~m}^{3} \mathrm{~kg}^{-1} \mathrm{~s}^{-2}$ at $68 \% \mathrm{CL}$, which is consistent with the value of CODATA 2018. However, the $H_{0}$ tension can't be solved with this method.

\section{Summary and discussion}

In this paper, we investigate how Newton's constant influences the CMB power spectra and the light curve of SINa. So the CMB data and SINa measurement can put a constraint on the Newton's constant. Combining the Planck data released in 2018, the BAO data and PANTHEON samples, we run CAMB and CosmoMC with a varying $G$ during the recombination epoch and the redshift ranges of SINa measurement. $G=G_{N}$ is located in the $68 \%$ CL ranges of the two periods. We find no evidence of the dynamic property of Newton's constant.

In addition, considering the effect of Newton's constant on the expansion history of our universe, we have a try to solve the $H_{0}$ tension by freeing $G$ based on the $\Lambda \mathrm{CDM}$ model. Adopting the combination of Planck 2018 TT,TE,EE+lowE+lensing+uncalibrated PANTHEON+ BAO, we obtain $\lambda_{i}=0.999 \pm 0.014$ and $H_{0}=67.62_{-1.25}^{+1.24}$ $\mathrm{km} \mathrm{s}^{-1} \mathrm{Mpc}^{-1}$ at $68 \% \mathrm{CL}$. With this method, the $H_{0}$ tension can't be solved. At the same time, our results show that Newton's constant from this model is consistent with the value given by CODATA 2018.

Acknowledgements We acknowledge the use of HPC Cluster of Tianhe II in National Supercomputing Center in Guangzhou and HPC Cluster of ITP-CAS. We would like to thank Qing-Guo Huang for his helpful discussions and advices on this paper.

Data Availability Statement This manuscript has no associated data or the data will not be deposited. [Authors' comment: We have shown all the nessesary data in the table and contour plot in our paper.]

Open Access This article is licensed under a Creative Commons Attribution 4.0 International License, which permits use, sharing, adaptation, distribution and reproduction in any medium or format, as long as you give appropriate credit to the original author(s) and the source, provide a link to the Creative Commons licence, and indicate if changes were made. The images or other third party material in this article are included in the article's Creative Commons licence, unless indicated otherwise in a credit line to the material. If material is not included in the article's Creative Commons licence and your intended use is not permitted by statutory regulation or exceeds the permitted use, you will need to obtain permission directly from the copyright holder. To view a copy of this licence, visit http://creativecomm ons.org/licenses/by/4.0/.

Funded by SCOAP S $^{3}$

\section{References}

1. G. Rosi, F. Sorrentino, L. Cacciapuoti, M. Prevedelli, G .M. Tino, Precision measurement of the Newtonian gravitational constant using cold atoms. Nature 510, 518 (2014). https://doi.org/10.1038/ nature13433. arXiv:1412.7954 [physics.atom-ph]

2. C. Umiltà, M. Ballardini, F. Finelli, D. Paoletti, JCAP 08, 017 (2015). https://doi.org/10.1088/1475-7516/2015/08/017. arXiv:1507.00718 [astro-ph.CO] 
3. M. Ballardini, F. Finelli, C. Umiltà, D. Paoletti, JCAP 05, 067 (2016). https://doi.org/10.1088/1475-7516/2016/05/067. arXiv:1601.03387 [astro-ph.CO]

4. O. Zahn, M. Zaldarriaga, Probing the Friedmann equation during recombination with future CMB experiments. Phys. Rev. D 67, 063002 (2003). https://doi.org/10.1103/PhysRevD.67.063002. arXiv:astro-ph/0212360

5. S. Galli, A. Melchiorri, G.F. Smoot, O. Zahn, From Cavendish to PLANCK: constraining Newton's gravitational constant with CMB temperature and polarization anisotropy. Phys. Rev. D 80, 023508 (2009). https://doi.org/10.1103/PhysRevD.80.023508. arXiv:0905.1808 [astro-ph.CO]

6. Y. Bai, J. Salvado, B.A. Stefanek, Cosmological constraints on the gravitational interactions of matter and dark matter. JCAP 1510, 029 (2015). https://doi.org/10.1088/1475-7516/2015/10/ 029. arXiv:1505.04789 [hep-ph]

7. S.S. Xue, Nucl. Phys. B 897, 326-345 (2015). https://doi.org/10. 1016/j.nuclphysb.2015.05.022. arXiv:1410.6152 [gr-qc]

8. C.J. Copi, A.N. Davis, L.M. Krauss, A new nucleosynthesis constraint on the variation of G. Phys. Rev. Lett. 92, 171301 (2004). https://doi.org/10.1103/PhysRevLett.92.171301. arXiv:astro-ph/0311334

9. J. Alvey, N. Sabti, M. Escudero, M. Fairbairn, Improved BBN constraints on the variation of the gravitational constant. Eur. Phys. J. C 80(2), 148 (2020). https://doi.org/10.1140/epjc/ s10052-020-7727-y. arXiv:1910.10730 [astro-ph.CO]

10. G.B. Gelmini, M. Kawasaki, A. Kusenko, K. Murai, V. Takhistov, Big Bang Nucleosynthesis constraints on sterile neutrino and lepton asymmetry of the Universe. (2020). [arXiv:2005.06721 [hep-ph]]

11. B.S. Wright, B. Li, Type Ia supernovae, standardizable candles, and gravity. Phys. Rev. D 97(8), 083505 (2018). https://doi.org/10. 1103/PhysRevD.97.083505. arXiv:1710.07018 [astro-ph.CO]

12. B.R. Zhang, M.J. Childress, T.M. Davis, N.V. Karpenka, C. Lidman, B.P. Schmidt, M. Smith, Mon. Not. R. Astron. Soc. 471(2), 2254-2285 (2017). https://doi.org/10.1093/mnras/ stx1600. arXiv:1706.07573 [astro-ph.CO]

13. S. Dhawan, S.W. Jha, B. Leibundgut, Astron. Astrophys. 609, A72 (2018). https://doi.org/10.1051/0004-6361/201731501. arXiv:1707.00715 [astro-ph.CO]

14. W. Zhao, B.S. Wright, B. Li, Constraining the time variation of Newton's constant $G$ with gravitational-wave standard sirens and supernovae. JCAP 1810, 052 (2018). https://doi.org/10.1088/ 1475-7516/2018/10/052. arXiv:1804.03066 [astro-ph.CO]

15. A. Riazuelo, J.P. Uzan, Cosmological observations in scalar-tensor quintessence. Phys. Rev. D 66, 023525 (2002). https://doi.org/10. 1103/PhysRevD.66.023525. arXiv:astro-ph/0107386

16. A. Vijaykumar, S.J. Kapadia, P. Ajith, Constraints on the time variation of the gravitational constant using gravitational wave observations of binary neutron stars. arXiv:2003.12832 [gr-qc]

17. M. Rossi, M. Ballardini, M. Braglia, F. Finelli, D. Paoletti, A.A. Starobinsky, C. Umiltà, Phys. Rev. D 100(10), 103524 (2019). https://doi.org/10.1103/PhysRevD.100.103524. arXiv: 1906.10218 [astro-ph.CO]

18. J. Solà Peracaula, A. Gomez-Valent, J. de Cruz Pérez, C. Moreno-Pulido, Brans-Dicke gravity with a cosmological constant smoothes out $\Lambda \mathrm{CDM}$ tensions. Astrophys. J. Lett. 886(1), L6 (2019). https://doi.org/10.3847/2041-8213/ab53e9. [arXiv: 1909.02554 [astro-ph.CO]]

19. J. Sola, A. Gomez-Valent, J. d. Perez, C. Moreno-Pulido, BransDicke cosmology with a $\Lambda$-term: a possible solution to $\Lambda \mathrm{CDM}$ tensions. (2020). [arXiv:2006.04273 [astro-ph.CO]]

20. N. Aghanim et al. (Planck), Planck 2018 results. VI. Cosmological parameters. arXiv:1807.06209 [astro-ph.CO]

21. D.M. Scolnic et al., The complete light-curve sample of spectroscopically confirmed SNe Ia from pan-STARRS1 and cosmological constraints from the combined pantheon sample. Astrophys.
J. 859(2), 101 (2018). https://doi.org/10.3847/1538-4357/aab9bb. arXiv:1710.00845 [astro-ph.CO]

22. A.G. Riess, S. Casertano, W. Yuan, L.M. Macri, D. Scolnic, Large magellanic cloud Cepheid standards provide a $1 \%$ foundation for the determination of the hubble constant and stronger evidence for physics beyond $\Lambda \mathrm{CDM}$. Astrophys. J. 876(1), 85 (2019). https://doi.org/10.3847/1538-4357/ab1422. arXiv:1903.07603 [astro-ph.CO]

23. M. Lattanzi, C. Burigana, M. Gerbino, A. Gruppuso, N. Mandolesi, P. Natoli, G. Polenta, L. Salvati, T. Trombetti, JCAP 02, 041 (2017). https://doi.org/10.1088/1475-7516/2017/02/041. arXiv:1611.01123 [astro-ph.CO]

24. Y. Huang, G.E. Addison, J.L. Weiland, C.L. Bennett, Astrophys. J. 869(1), 38 (2018). https://doi.org/10.3847/1538-4357/aaeb1f. arXiv: 1804.05428 [astro-ph.CO]

25. D.N. Spergel, R. Flauger, R. Hložek, Phys. Rev. D 91(2), 023518 (2015). https://doi.org/10.1103/PhysRevD.91.023518. arXiv: 1312.3313 [astro-ph.CO]

26. C. Bennett, D. Larson, J. Weiland, G. Hinshaw, Astrophys. J. 794, 135 (2014). https://doi.org/10.1088/0004-637X/794/2/135. arXiv: 1406.1718 [astro-ph.CO]

27. L. Chen, Q.G. Huang, K. Wang, Eur. Phys. J. C 77(11), 762 (2017). https://doi.org/10.1140/epjc/s10052-017-5344-1. arXiv:1707.02742 [astro-ph.CO]

28. S. Vagnozzi, New physics in light of the $H_{0}$ tension: an alternative view. (2019). [arXiv:1907.07569 [astro-ph.CO]]

29. E. Di Valentino, A. Melchiorri, J. Silk, Phys. Lett. B 761, 242-246 (2016). https://doi.org/10.1016/j.physletb.2016.08.043. arXiv:1606.00634 [astro-ph.CO]

30. C.D. Kreisch, F.Y. Cyr-Racine, O. Doré, The neutrino puzzle: anomalies, interactions, and cosmological tensions. (2019). [arXiv:1902.00534 [astro-ph.CO]]

31. L. Hart, J. Chluba, Mon. Not. R. Astron. Soc. 493(3), 3255-3263 (2020). https://doi.org/10.1093/mnras/staa412. arXiv:1912.03986 [astro-ph.CO]

32. M. Ballardini, M. Braglia, F. Finelli, D. Paoletti, A.A. Starobinsky, C. Umiltà, Scalar-tensor theories of gravity, neutrino physics, and the $H_{0}$ tension. (2020). [arXiv:2004.14349 [astro-ph.CO]]

33. E. Di Valentino, A. Melchiorri, E.V. Linder, J. Silk, Phys. Rev. D 96(2), 023523 (2017). https://doi.org/10.1103/PhysRevD.96. 023523. arXiv:1704.00762 [astro-ph.CO]

34. W. Yang, S. Pan, E. Di Valentino, E.N. Saridakis, S. Chakraborty, Phys. Rev. D 99(4), 043543 (2019). https://doi.org/10.1103/ PhysRevD.99.043543. arXiv:1810.05141 [astro-ph.CO]

35. X.D. Li, C.G. Sabiu, C. Park, Y. Wang, G b Zhao, H. Park, A. Shafieloo, J. Kim, S.E. Hong, Astrophys. J. 856(2), 88 (2018). https://doi.org/10.3847/1538-4357/aab42e. arXiv:1803.01851 [astro-ph.CO]

36. X. Zhang, Q.G. Huang, Sci. China Phys. Mech. Astron. 63(9), 290402 (2020). https://doi.org/10.1007/s11433-019-1504-8. arXiv:1911.09439 [astro-ph.CO]

37. G.B. Zhao, M. Raveri, L. Pogosian, Y. Wang, R.G. Crittenden, W.J. Handley, W.J. Percival, F. Beutler, J. Brinkmann, C.H. Chuang, A.J. Cuesta, D.J. Eisenstein, F.S. Kitaura, K. Koyama, B. L'Huillier, R.C. Nichol, M.M. Pieri, S. Rodriguez-Torres, A.J. Ross, G. Rossi, A.G. Sánchez, A. Shafieloo, J.L. Tinker, R. Tojeiro, J.A. Vazquez, H. Zhang, Nat. Astron. 1(9), 627-632 (2017). https://doi.org/10. 1038/s41550-017-0216-z. arXiv:1701.08165 [astro-ph.CO]

38. Q.G. Huang, K. Wang, Eur. Phys. J. C 76(9), 506 (2016). https://doi. org/10.1140/epjc/s10052-016-4352-x. arXiv:1606.05965 [astroph.CO]

39. V. Poulin, T.L. Smith, T. Karwal, M. Kamionkowski, Phys. Rev. Lett. 122(22), 221301 (2019). https://doi.org/10.1103/ PhysRevLett.122.221301. arXiv:1811.04083 [astro-ph.CO] 
40. T.L. Smith, V. Poulin, M.A. Amin, Phys. Rev. D 101(6), 063523 (2020). https://doi.org/10.1103/PhysRevD.101.063523. arXiv:1908.06995 [astro-ph.CO]

41. T. Karwal, M. Kamionkowski, Phys. Rev. D 94(10), 103523 (2016). https://doi.org/10.1103/PhysRevD.94.103523. arXiv:1608.01309 [astro-ph.CO]

42. M. Braglia, W.T. Emond, F. Finelli, A.E. Gumrukcuoglu, K. Koyama, Unified framework for Early Dark Energy from $\alpha$ attractors. (2020). [arXiv:2005.14053 [astro-ph.CO]]

43. D. Bégué, C. Stahl, S.S. Xue, Nucl. Phys. B 940, 312 320 (2019). https://doi.org/10.1016/j.nuclphysb.2019.01.001. arXiv:1702.03185 [astro-ph.CO]

44. S. Kumar, R.C. Nunes, Phys. Rev. D 94(12), 123511 (2016). https:// doi.org/10.1103/PhysRevD.94.123511. arXiv:1608.02454 [astroph.CO]

45. W. Yang, S. Pan, E. Di Valentino, R.C. Nunes, S. Vagnozzi, D.F. Mota, JCAP 09, 019 (2018). https://doi.org/10.1088/1475-7516/ 2018/09/019. arXiv:1805.08252 [astro-ph.CO]

46. E. Di Valentino, A. Melchiorri, O. Mena, Phys. Rev. D 96(4), 043503 (2017). https://doi.org/10.1103/PhysRevD.96. 043503. arXiv:1704.08342 [astro-ph.CO]

47. E. Mörtsell, S. Dhawan, JCAP 09, 025 (2018). https://doi.org/10. 1088/1475-7516/2018/09/025. arXiv:1801.07260 [astro-ph.CO]

48. P. Ko, Y. Tang, Phys. Lett. B 762, 462-466 (2016). https://doi.org/ 10.1016/j.physletb.2016.10.001. arXiv:1608.01083 [hep-ph]

49. P. Agrawal, F.Y. Cyr-Racine, D. Pinner, L. Randall, Rock 'n' Roll Solutions to the Hubble Tension. (2019). [arXiv:1904.01016 [astroph.CO]]
50. M.X. Lin, G. Benevento, W. Hu, M. Raveri, Phys. Rev. D 100(6), 063542 (2019). https://doi.org/10.1103/PhysRevD.100. 063542. arXiv:1905.12618 [astro-ph.CO]

51. G. Ballesteros, A. Notari, F. Rompineve, The $H_{0}$ tension: $\Delta G_{N}$ vs. $\Delta N_{\text {eff }} \cdot \operatorname{arXiv}: 2004.05049$ [astro-ph.CO]

52. M. Braglia, M. Ballardini, W.T. Emond, F. Finelli, A.E. Gumrukcuoglu, K. Koyama, D. Paoletti, A larger value for $H_{0}$ by an evolving gravitational constant. arXiv:2004.11161 [astro-ph.CO]

53. A. Lewis, S. Bridle, Cosmological parameters from CMB and other data: a Monte Carlo approach. Phys. Rev. D 66, 103511 (2002). arXiv:astro-ph/0205436

54. D.J. Eisenstein, W. Hu, Baryonic features in the matter transfer function. Astrophys. J. 496, 605 (1998). https://doi.org/10.1086/ 305424. arXiv:astro-ph/9709112

55. S. Seager, D.D. Sasselov, D. Scott, A new calculation of the recombination epoch. Astrophys. J. 523, L1 (1999). https://doi.org/10. 1086/312250. arXiv:astro-ph/9909275

56. A .J. Ross, L. Samushia, C. Howlett, W .J. Percival, A. Burden, M. Manera, The clustering of the SDSS DR7 main Galaxy sample-I. A 4 per cent distance measure at $z=0.15$. Mon. Not. R. Astron. Soc. 449(1), 835 (2015). arXiv:1409.3242 [astro-ph.CO]

57. F. Beutler et al., The $6 \mathrm{dF}$ galaxy survey: baryon acoustic oscillations and the local hubble constant. Mon. Not. R. Astron. Soc. 416, 3017 (2011). arXiv:1106.3366 [astro-ph.CO]

58. S. Alam et al. (BOSS Collaboration), The clustering of galaxies in the completed SDSS-III Baryon Oscillation Spectroscopic Survey: cosmological analysis of the DR12 galaxy sample. Mon. Not. R. Astron. Soc. 470(3), 2617 (2017). arXiv:1607.03155 [astro-ph.CO] 